\section{On the evidence for ESR 1 amplification in breast cancer}

Frederik Holst, Cathy B. Moelans, Martin Filipits, Christian F. Singer, Ronald Simon and Paul J. van Diest

We read the correspondence regarding the recent Review (The different roles of ER subtypes in cancer biology and therapy. Nature Rev. Cancer 11, 597-608 (2011)) ${ }^{1}$ between Ke-Da Yu and Zhi-Ming Shao and the authors of the Review with great interest ${ }^{1-3}$. The ongoing debate concerning the prevalence and the clinical relevance of oestrogen receptor 1 (ESR1) amplification reflects the highly visible evidence for this genetic aberration despite the challenging data.

There are now three studies from three different groups, which all confirm ESR1 amplification in $20.6 \%, 22.6 \%$ and $21.6 \%$ of analysed breast cancers by fluorescence in situ hybridization (FISH $)^{4-6}$, including one study by Tsiambas et al. ${ }^{6}$ that is only rarely cited. Likewise, it has hardly been recognized that Moelans et al. ${ }^{7}$ detected $16 \%$ and $20 \%{ }^{7,8}$ ESR1 (mostly low level) gains and amplifications in two independent multiplex ligation-dependent probe amplification (MLPA) studies, which is substantially higher than the $8 \%{ }^{9}$ cited by Thomas and Gustafsson ${ }^{3}$ and which may be related to the different patient sets in their three publications. Using a cut-off of 2 (for the grade of copy number imbalance) instead of $>2$, Nessling et al. ${ }^{10}$ would have found around $19 \%$ instead of $10 \%$ amplification by array comparative genomic hybridization $(\mathrm{aCGH})^{10}$, illustrating that minor changes in the threshold criteria may have major effects on the outcome of ESR1 studies. There are five studies by four different groups using DNA-specific methods that are independent of observer subjectivity, such as Southern blot, aCGH or MLPA PCR, which have found rates of $10 \%$ and greater ESR 1 amplification ${ }^{7,8,10,11}$ or increased copy number ${ }^{7,8,12}$ in breast cancer. Including the $20 \%$ increased ESR 1 copy numbers in oestrogen receptor (ER)-positive tumours reported by Dunbier et al. ${ }^{12}$, these studies typically reported low-level copy number gains. It is easily imaginable that there could be copy number gains both just above and just below the detection level and within the background of measurement.
When non-morphological methods are used, contamination with non-tumour cells (which could be resolved by laser microdissection) and intermingled mosaic-patterned intratumoural heterogeneity of copy number status - as also shown in case of ERBB2 (REFS 13,14) - could obscure the presence of low-level amplification, although this may be readily picked up by fluorescence in situ hybridization (FISH).

Evidence of correlation between elevated ESR1 copy numbers and high ER expression was found in five studies from five independent groups ${ }^{4-6,9,12}$, raising the question of whether ESR 1 copy numbers could be a better clinical marker for response to hormone therapy than protein expression. In their recent ESR1 study, Ejlertsen et al. ${ }^{15}$ did not reproduce previously claimed therapeutic resistance of tumours with amplified ESR1, and there was no difference in distribution in the percentage of ER-expressing tumour cells between tumours with amplified ESR1 $\left(13.6 \% \mathrm{ER}^{+}\right)$and those of normal copy number $\left(82.2 \% \mathrm{ER}^{+}\right)$. However, they found significantly lower ER expression in ESR1-deleted tumours ${ }^{15,16}$.

In summary, there is growing evidence that ESR1 gain or amplification is a fairly frequent event in breast cancer, although it is difficult to detect with current analysis and threshold techniques, which were initially established for ERBB2 measurement. The clinical relevance of ESR 1 gains and amplifications in the sense of prognostic value and prediction of response to hormonal treatment requires further studies, similar to earlier debates for ERBB2 amplification.

Frederik Holst and Ronald Simon are at the Department of Pathology, University Medical Center Hamburg-Eppendorf, Martinistrasse 52, 20246 Hamburg, Germany.

Cathy B. Moelans and Paul J. van Diest are at the Department of Pathology, University Medical Center Utrecht, Heidelberglaan 100, 3584 CX Utrecht, The Netherlands.
Martin Filipits is at the Institute of Cancer Research, Department of Medicine I, Medical University of Vienna, Borschkegasse 8a, 1090 Vienna, Austria.

Christian F. Singer is at the Department of Special Gynecology, Medical University of Vienna, Währinger Gürtel 18-20, 1090 Vienna, Austria.

Correspondence to F.H. e-mail:f.holst@uke.de

doi: 10.1038/nrc3093-c3

1. Thomas, C. $\&$ Gustafsson, J.-A. The different roles of ER subtypes in cancer biology and therapy. Nature Rev. Cancer 11, 597-608 (2011).

2. Yu, K.-D. \& Shao, Z.-M. ESR 1 gene amplification: another mechanism regulating the cellular levels of ERa. Nature Rev. Cancer 11, 823 (2011).

3. Thomas, C. \& Gustafsson, J.-A. Not enough evidence to include ESR 1 amplification. Nature Rev. Cancer 11 , 823 (2011).

4. Holst, F. et al. Estrogen receptor alpha (ESR1) gene amplification is frequent in breast cancer. Nature Genet. 39, 655-660 (2007).

5. Tomita, S. et al. Estrogen receptor a gene ESR 1 amplification may predict endocrine therapy responsiveness in breast cancer patients. Cancer Sci. 100, 1012-1017 (2009).

6. Tsiambas, E. et al. Significance of estrogen receptor 1 (ESR-1) gene imbalances in colon and hepatocellular carcinomas based on tissue microarrays analysis. Med. Oncol. 28, 934-940 (2011).

7. Moelans, C. B. et al. Molecular profiling of invasive breast cancer by multiplex ligation-dependent probe amplification-based copy number analysis of tumor suppressor and oncogenes. Mod. Pathol. 23, 1029-1039 (2010).

8. Moelans, C. B. et al. Molecular differences between ductal carcinoma in situ and adjacent invasive breast carcinoma: a multiplex ligation-dependent probe amplification study. Anal. Cell Pathol. (Amst) 33 165-173 (2010)

9. Moelans, C. B. et al. ESR1 amplification is rare in breast cancer and is associated with high grade and high proliferation: A multiplex ligation-dependent probe amplification study. Cell Oncol. 34, 489-494 (2011).

10. Nessling, M. et al. Candidate genes in breast cancer revealed by microarray-based comparative genomic hybridization of archived tissue. Cancer Res. 65, 439-447 (2005)

11. Nembrot, M., Quintana, B. \& Mordoh, J. Estrogen receptor gene amplification is found in some estrogen receptor-positive human breast tumors. Biochem. Biophys. Res. Commun. 166, 601-607 (1990).

12. Dunbier, A. K. et al. ESR 1 is co-expressed with closely adjacent uncharacterised genes spanning a breast cancer susceptibility locus at 6q25.1. PLoS Genet. 7, e 1001382 (2011).

13. Szollosi, J. et al. ERBB-2 (HER2/neu) gene copy number, p185HER-2 overexpression, and intratumor heterogeneity in human breast cancer. Cancer Res. 55, 5400-5407 (1995)

14. Bernasconi, B. et al. Genetic heterogeneity in HER2 testing may influence therapy eligibility. Breast Cancer Res. Treat 8 Sep 2011 (doi:10.1007) s10549-011-1744-3).

15. Ejlertsen, B. et al. Prognostic and predictive role of ESR1 status for postmenopausal patients with endocrine-responsive early breast cancer in the Danish cohort of the BIG 1-98 trial. Ann. Oncol. 10 Oct 2011 (doi: 10.1093/annonc/mdr438).

16. Vang Nielsen, K. et al. Amplification of ESR1 may predict resistance to adjuvant tamoxifen in postmenopausal patients with hormone receptor positive breast cancer. Breast Cancer Res. Treat 127 , 345-355 (2011).

\section{Competing interests statement}

The University Medical Center Hamburg-Eppendorf has filed a patent application for certain technologies related to this correspondence. 